\title{
Vertexing Software and Methods for the ILC
}

\author{
Georgios Voutsinas*广 \\ DESY \\ E-mail: georgios.voutsinasdesy.de
}

The International Linear Collider (ILC) is a proposed linear electron-positron collider. Its beam energy will be tunable, featuring a $\sqrt{s}$ from $250 \mathrm{GeV}$ to $500 \mathrm{GeV}$, which will be possibly upgraded to $1 \mathrm{TeV}$. It is going to be a high precision machine, featuring a well defined initial state, clean final state and triggerless operation of its detectors. Also, both electron and positron beams will be polarised, at a level of $80 \%$ and $30 \%$ (at least) respectively. It will be equipped with two multipurpose detectors, the International Large Detector (ILD) and the Silicon Detector (SiD) [1]. The two detectors will share the same experimental area. They will take data alternatively, according to a push-pull scheme. To achieve the ILC physics goals, unprecedented momentum, jet energy and impact parameter resolution are required for the ILC detectors.

The identification of the jet's flavour plays a central role in ILC physics program. Excellent tracking and vertexing performance is required in order to distinguish $\mathrm{b}, \mathrm{c}$ and light quark jets. In these proceedings, we will review the foreseen strategies for tracking, vertexing and flavour tagging in the ILC detectors. Various methods for pattern recognition in tracking exist, depending on the detector design and/or its technology. On the other hand, both ILD and SiD share the same tools for vertexing and flavour tagging.

The 23rd International Workshop on Vertex Detectors,

15-19 September 2014

Macha Lake, The Czech Republic

\footnotetext{
* Speaker.

†n behalf of the ILD and the SiD groups
} 


\section{The ILD and the SiD detectors}

The designs of ILD and SiD have been optimised according to the particle flow paradigm. The particle flow aims to individually reconstruct every particle of an event. The two detectors follow the basic layout of the modern HEP detectors. Going outwards from the interaction point (IP), one will meet the vertex detector (VXD hereafter), the main tracker and the electromagnetic and hadronic calorimeters. Both calorimeters are located inside the coil, in order to optimise the jet energy resolution. An iron yoke, equipped with muons chambers, surrounds the coil. Hermeticity is also crucial: the forward region is instrumented with tracking disks and calorimeters. ILD provides satisfactory tracking and calorimetric coverage down to $\sim \theta=10^{0}$ and $\theta=13^{0}$ respectively $(\theta$ denotes the polar angle). The $\mathrm{SiD}$ tracking system can provide at least 6 hits down to $\theta=8^{0}$.

The main difference between ILD and SiD lays in the main tracker; ILD has a Time Projection Chamber (TPC), which can provide up to 220 space-points, being complemented by two internal (Silicon Inner Tracker - SIT) and one external (Silicon External Tracker - SET) silicon strip detector layers; SiD features an all-silicon tracker, which consists of a pixelated VXD and a tracker having 5 layers and 4 disks of single sided silicon strip layers.

\subsection{ILC vertex detectors}

The ILC physics program requires a VXD that exhibits excellent performance in terms of flavour tagging, meaning b- and c-jet identification, and track reconstruction. Flavour tagging is essential for numerous studies, the most prominent being the precise measurement of the Higgs boson couplings to the fermions. The key for the flavour tagging is the efficient reconstruction of displaced vertices. To do so, one has to provide a detector that exhibits high spatial resolution, while featuring very low material budget, and located as close as possible to the IP.

The running constraints of the ILC environment play a major role in the VXD design. They are mostly defined by the beam related background due to beamstrahlung [2]. Experience acquired in previous experiments and intuition indicate us that the pixel occupancy should be maintained below $\sim 1 \%$. The beam induced background dictates also the sensor's radiation tolerance. However, the latter is considered rather moderate for the ILC environment.

The ILD VXD is composed of ladders, arranged in concentric cylindrical layers to form barrels. There are two main VXD candidate geometries, which are illustrated in Figure 1. One is composed of 5 single-sided layers, meaning that each layer is equipped with silicon pixel sensors mounted on one side of a light mechanical support. The other consists of 3 double-sided layers, meaning that silicon pixel sensors will equip both sides of the mechanical support, with a $2 \mathrm{~mm}$ spacing between them, providing this way two very high precision measurements per layer. In these proceedings, we will examine only the double layers option.

The SiD VXD consists of 5 layers in the barrel part and 4 inner and 3 outer pixelated disks in the forward region, see Figure 1. SiD collaboration considers sensor technologies, that can offer single bunch crossing time resolution: this way, the effect of the beam background will be significantly suppressed. 

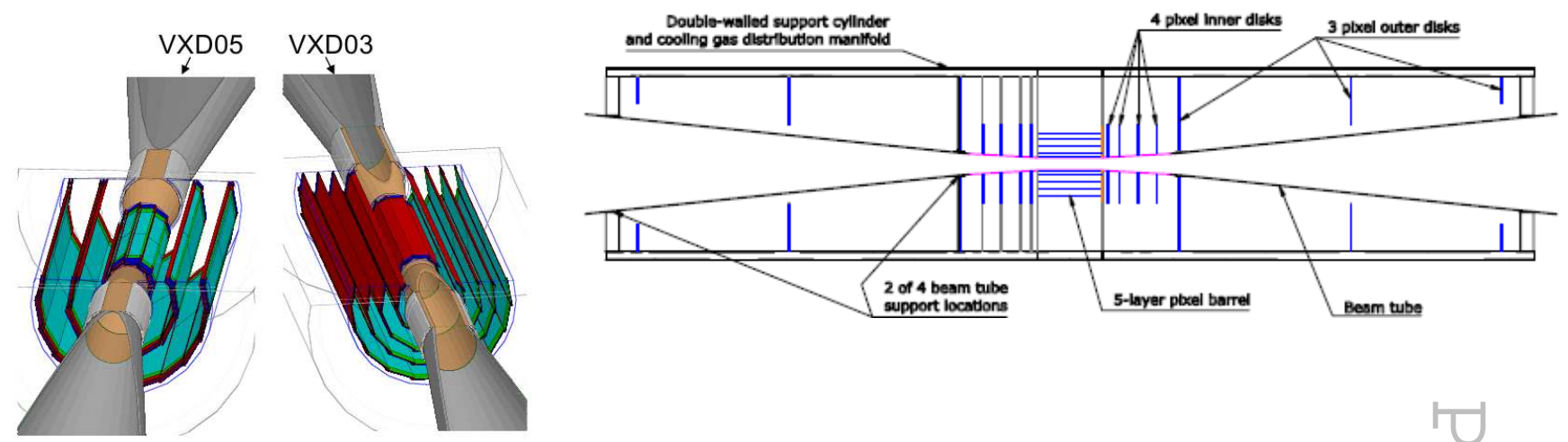

Figure 1: Left plot, ILD VXD geometries: 5 single layers (VXD03, right) and 3 double layers (VXD05, left). Right plot: SID VXD layout.

\section{Tracking}

The strategies of the ILC experiments for track finding are going to be discussed in this section. A variety of approaches for pattern recognition has been explored, depending on the nature and technology of the tracking system.

\subsection{Pattern recognition in ILD}

The ILD has 3 tracking subsystems, the TPC, the VXD-SIT and the Forward Tracking Disks (FTD), capable to provide standalone track reconstruction. The pattern recognition follows this approach; track reconstruction takes place independently in each subsystem. We will briefly describe the pattern recognition in each subdetector.

The TPC uses the Clupatra track reconstruction processor. Clupatra starts by forming seeds at the outer pad rows of the TPC, by using a nearest neighbour hit clustering algorithm. The seeds are propagated inwards, to the next pad row, using a Kalman filter. In each pad row, the most compatible hit (by the means of a $\chi^{2}$ criterio) is associated to the track. The track state is updated and the track is propagated to the next pad row, where this procedure is repeated.

The VXD-SIT detectors are divided into stereo angle sectors. A brute force triplet search, based on a set of seed layers, takes place in each angular sector. A helix is fitted to each seed triplet, and then it is extrapolated inwards. Hits are attached according to their distance from the track helix. Finally, the track is refitted using a Kalman fitter.

The pattern recognition at the FTD is based on a cellular automaton algorithm [6]. A Kalman fitter is used for track fitting.

Finally, the track segments reconstructed in each sub-detector are merged to produce the final track. The merging is based on parameter compatibility. A comprehensive up to date description of the ILD tracking software, the pattern recognition methods used in the tracking subsystems as well as its performance is given in [3]. The track finding efficiency of ILD reaches or exceeds $99.7 \%$ for particles having $P_{T} \geq 1 \mathrm{GeV}$ for the whole polar angle spectrum, while it is higher than $99.8 \%$ for $|\cos \theta|<0.95$.

A lower performance is observed for low $P_{T}$ tracks, which can be recovered by optimising the standalone silicon tracking. Indeed, we have observed that the standard silicon tracking used 
for the Detailed Baseline Design (DBD) studies doesn't show a satisfactory performance under realistic conditions (Figure 2). Realistic conditions means when the beam induced background is taken into account, which means that the pattern recognition algorithms should deal with $O\left(10^{5}\right)$ $O\left(10^{6}\right)$ hits at the VXD. There are two new approaches currently under investigation. One is the FPCCD track finder, which is documented in [4]. It has been observed that it significantly increases the tracking performance, for low $P_{T}$ tracks. Here we will focus on the second approach, a cellular automaton algorithm based on mini-vectors.
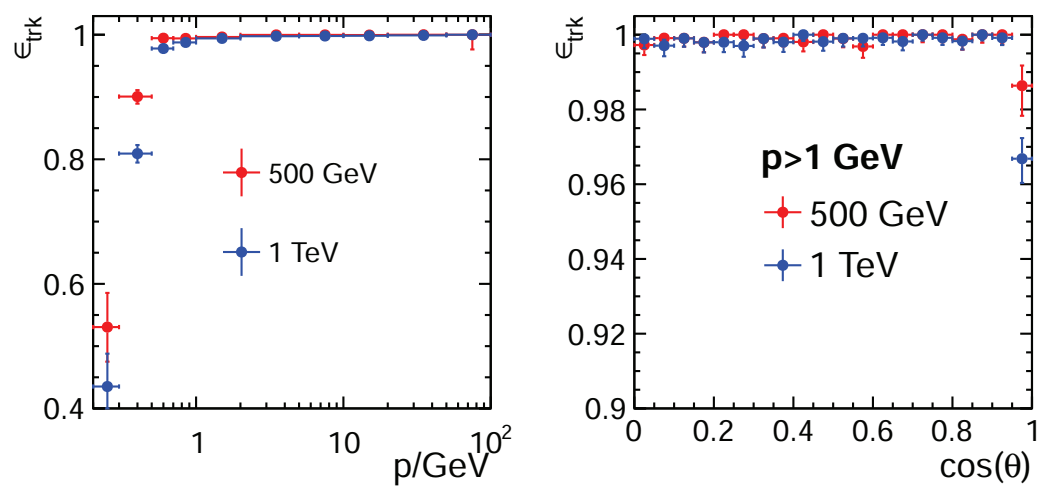

Figure 2: Tracking efficiency as a function of momentum (left) and of the cosine of the polar angle (right) for $t \bar{t} \rightarrow 6$ jets events at $\sqrt{s} 500 \mathrm{GeV}$ and $1 \mathrm{TeV}$. (from[1])

The use of mini-vectors is motivated from the double sided structure of the VXD ladders. The hits generated on the two sides by a traversing particle could be correlated to construct a minivector. A tracking based on mini-vectors is appealing in particular for a CMOS VXD design, where one side will be equipped with highly segmented square pixels aiming for high spatial resolution, while on the other side the pixels will be elongated in the column direction aiming for high time resolution [5]. Thus one can timestamp the mini-vector, using the time information from the hit on the fast layer, while preserving the spatial resolution.

We use the following method to construct the mini-vectors. As a first step, in order to reduce the combinatorics, the VXD is divided into stereo angle sectors. In each sector, we create all the possible hit pairs at every VXD double layer. We examine if the $\delta \theta$ ( $\theta$ stands for the polar angle of each hit) between the hits of a pair is below a maximum value. If yes, we form a mini-vector out of this pair of hits. The maximum $\delta \theta$ value has been defined by studying simulated $t \bar{t}$ events, where we have used the Monte-Carlo information in order to find relations between hits in adjacent layers belonging to the same track. After constructing all the candidate mini-vectors, we try to connect them into tracks. A cellular automaton is used to connect the mini-vectors of neighbouring sectors. The core tools of the cellular automaton are already a part of the ILD reconstruction software; they are used for the standalone tracking at the FTD [6]. The criteria, applied by the cellular automaton, whether to connect two pairs of mini-vectors to form a track segment are mainly based on the relative angles between them.

The mini-vector based tracking has been studied versus a number of CMOS based VXD designs. We used a $t \bar{t}$ sample at $\sqrt{s} 500 \mathrm{GeV}$ where the pair background has been overlayed accordingly to each layer's time resolution. The considered CMOS VXD designs are summarised in Table 
1. The performance, in terms of tracking efficiency, "bad" track reconstruction rate and CPU time has been evaluated. The examined track sample, used to evaluate the efficiency, consists of all charged particles inside the geometrical acceptance of the detector, which are not decayed inside the tracker. A particle is denoted as found if: a) at least $75 \%$ of the hits of the corresponding track arise from the same particle and b) at least 4 VXD hits are associated with the track (criterion valid only for tracks that cross all the VXD layers). The efficiency is illustrated in Figure 3 . We observe a striking improvement in the region below $1 \mathrm{GeV}$.

\begin{tabular}{|c|c|c|c|c|c|c|c|}
\hline \multicolumn{2}{|c|}{} & \multicolumn{2}{l|}{ CMOS VXD1 } & \multicolumn{2}{l|}{ CMOS VXD2 } & \multicolumn{2}{l|}{ CMOS VXD3 } \\
\hline Layer & Radius $(\mathrm{mm})$ & $\mathrm{SR}(\mu \mathrm{m})$ & $\mathrm{TR}(\mu s)$ & $\mathrm{SR}(\mu m)$ & $\mathrm{TR}(\mu s)$ & $\mathrm{SR}(\mu m)$ & TR $(\mu s)$ \\
\hline DL1 & $16 / 18$ & $3 / 6$ & $50 / 2$ & $5 / 5$ & $8 / 8$ & $3 / 5$ & $50 / 8$ \\
DL2 & $37 / 39$ & $4 / 10$ & $100 / 7$ & $5 / 5$ & $16 / 16$ & $5 / 5$ & $16 / 16$ \\
DL3 & $58 / 60$ & $4 / 10$ & $100 / 7$ & $5 / 5$ & $16 / 16$ & $5 / 5$ & $16 / 16$ \\
\hline
\end{tabular}

Table 1: Studied CMOS based VXD model. The spatial (SR) and time (TR) resolutions per layer are stated.

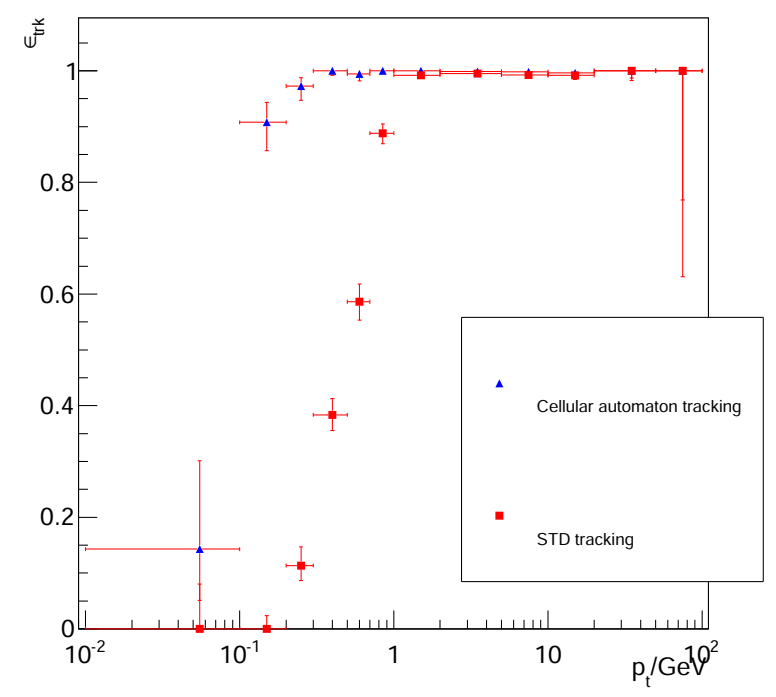

Figure 3: Track finding efficiency as a function of $P_{T}$ for the CMOS VXD1 design. Red full squares: efficiency evaluated using the standard ILD tracking (the one used for the DBD studies). Blue full triangles: efficiency evaluated when the standard ILD silicon tracking has been replaced by a mini-vector based cellular automaton tracking.

Table 2 states the CPU performance and bad track reconstruction rate of the algorithm for the studied VXD designs. We observe that a fast second layer (CMOS VXD1 design) improves significantly the performance, even if the overall number of hits that the algorithm has to deal with is higher.

\footnotetext{
${ }^{1}$ As bad tracks we define tracks that are either ghosts, or coming from beam background particles
} 


\begin{tabular}{|c|c|c|c|}
\hline & CMOS VXD1 & CMOS VXD2 & CMOS VXD3 \\
\hline Total hits $\left(\times 10^{3}\right)$ & 120 & 30 & 100 \\
Bad tracks rate & 56 & 84 & 100 \\
Time/evt $(\mathrm{s})$ & 15 & 10 & 100 \\
\hline
\end{tabular}

Table 2: Total number of hits per readout frame in VXD, number of reconstructed bad tracks per event and processing time required per event for various studied CMOS VXD designs.

\subsection{Pattern recognition in $\mathrm{SiD}$}

The pattern recognition in $\mathrm{SiD}$ is based on a track following approach. A strategy builder defines various combinations for the seeding, the confirmation and the extension layers. The first step is the seeding: as candidate seeds are considered all 3-hit combinations at the 3 seeding layers. The two innermost VXD layers are not participating at the seeding, in order to mitigate the combinatorics due to the beam background induced hits. The 3-hit combinations are examined if they fulfill the helix hypothesis. If yes, the helix is then extrapolated to the confirmation layer, where the presence of a compatible hit is required. For each candidate hit, a helix fit is performed. If its $\chi^{2}$ value is below the defined threshold, the candidate is accepted. In case that a matching hit cannot be found at the confirmation layer, the track candidate is rejected. Thus the combinatorial background is kept under control. Then the track is extrapolated to the extension layers, and the track-hit association procedure is repeated. The tracks with less than 7 hits are discarded. In case of hit sharing between tracks, only the best track candidate is kept. The best candidate is defined as the longest track. When the tracks have the same number of hits, the one with lower $\chi^{2}$ is considered as the best.

The track reconstruction follows an inside-out approach. To avoid losing tracks with a large impact parameter, which will not create hits at the inner tracker layers, a calorimeter assisted tracking algorithm has been developed [7]. In the framework of the DBD studies, the SID tracking efficiency has been evaluated with a Z' sample of $1 \mathrm{TeV}$ mass, including beam induced background. The average track finding efficiency was found $\sim 98 \%$ [1].

\section{Vertexing}

The first vertexing-flavour tagging tool for ILC was the LCFIVertex software package [8], which has been used for physics studies intented for the ILD and the SiD Letter of Intent (LoI). The software currently in use in ILC is the LCFIPlus [ [ [ ], an improvement and extension of the LCFIVertex. The main difference is that the LCFIVertex applies a jet-based vertexing, which means that the jet clustering precede the vertexing, while LCFIPlus performs an event-based vertexing and then proceeds to jet clustering. The LCFIPlus approach was observed to be superior, especially in many-jet events.

Information stemming from secondary IP vertices which arise from heavy flavour decays, is crucial in order to identify the jet's flavour. Therefore, vertices that do not originate from heavy flavour decays should be properly identified and not enter into the jet flavour identification procedure, which is described below. The origin of such vertices can be photon conversions in the 
material of the detector or $\mathrm{K} / \Lambda$ decays. An appropriate track selection, after the standard track reconstruction and before flavour tagging, will suppress the number of such vertices that enter into the jet flavour identification procedure.

\subsection{LCFIVertex - ZVTOP algorithm}

The vertexing in the LCFIVertex software is based on a re-implementation of the topological vertex finder of the SLAC Large Detector (SLD) experiment, ZVTOP [10]. The ZVTOP software package contains two complementary algorithms for topological vertex reconstruction, called ZVRES and ZVKIN. ZVRES is used to reconstruct multiprong vertices while ZVKIN is specialised for the case of one prong vertices. Here we are going to give a brief description of the ZVRES algorithm. The main idea of the algorithm is to construct a function $V(\vec{r})$, which describes the vertex probability at the position $\vec{r}$. In order to construct this function, each track is ascribed a probability tube in space, obtained from the errors on the track helix parameters, see left part of Figure $\bigoplus$.
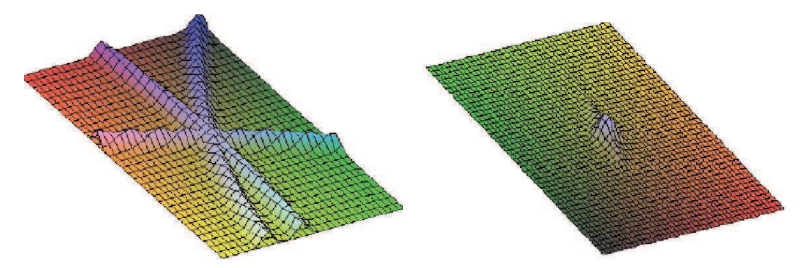

Figure 4: Left: the track density function and right: the vertex density function, projected on the xy plane. Image taken from [11].

The basic vertex density function is derived by combining the track probability functions. It will acquire high values in space regions where two or more tracks overlap, see right part of Figure 4. Out of the vertex density a number of secondary and tertiary vertices can be reconstructed. The heavy hadrons are expected to decay close to the jet axis; therefore, the vertex functions is appropriately weighed outside a cylinder of $50 \mu \mathrm{m}$ radius around the jet axis.

\subsection{LCFIPlus software package}

The main motivation to develop an event-based vertexing is the possibility of tracks from the same secondary vertex to be assigned to different jets, thus significantly decreasing the vertex reconstruction efficiency. To avoid this problem, the vertex reconstruction is performed prior to jet clustering. The reconstructed vertices are then associated intact to jets. Thus, the ZVTOP algorithm can't be used for vertex reconstruction, since it makes use of jet information. Therefore, new algorithms have been developed.

For the reconstruction of the primary vertex, a tear down algorithm was developed. All the tracks that satisfy the selection criteria (relatively small impact parameters in $R \phi$ plane and $\mathrm{Z}$ direction and a minimum number of hits in the VXD-FTD) are fitted to a common vertex. The track featuring the highest $\chi^{2}$ is removed and the vertex is refitted, until a specified $\chi^{2}$ threshold is reached. The remaining tracks are removed from the track sample that will be used for the secondary vertices reconstruction. 
The reconstruction of secondary vertices takes place via a build-up type algorithm. The first step is the creation of all possible pairs of tracks. Then it tries to fit them to their common vertex. The common vertex of a pair of tracks is defined as their point of closest approach. If the fit is successful, more tracks are tested if they can be associated to this vertex.

\section{Flavour tagging}

The vertex reconstruction is followed by the jet clustering. As was mentioned, the reconstructed vertices are associated intact within the jets. The ILC experiments use the boosted decision trees (BDTs) based heavy flavour tagging developed by the LCFIPlus collaboration. One of its main features is that it uses a different set of input variables for the BDTs, depending on the jet's vertex multiplicity. Therefore, after the jet clustering, the jets are separated to the following 4 classes: the first class contains jets that no secondary vertex has been associated with them. The second class contains jets with one secondary vertex. The third class is formed by jets which have one secondary vertex plus a track with high impact parameter. Finally, the fourth class corresponds to jets with two or more secondary vertices. An illustration of the various jet classes is given in Figure 5.
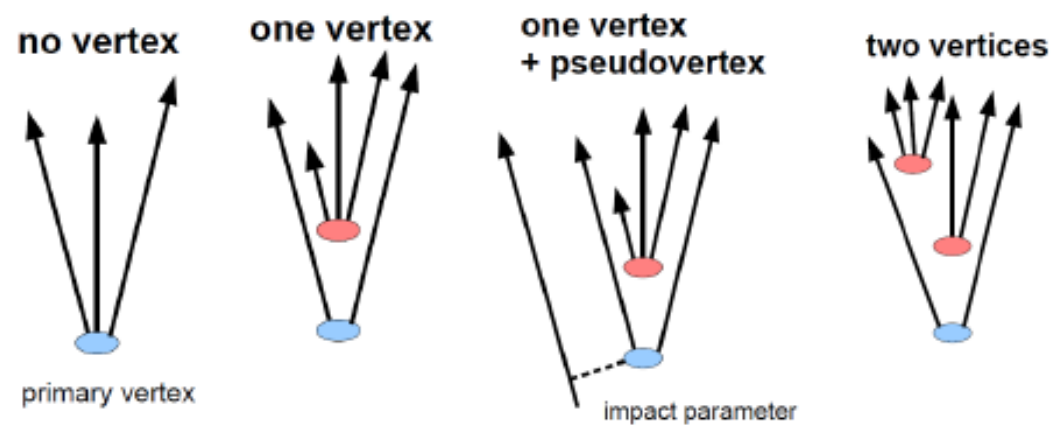

Figure 5: Illustration of the various jet classes according to the number of the secondary vertices. Image taken from P. Roloff.

When a secondary vertex has been found, we try to exploit the displaced vertex information. Very important for the $\mathrm{b}-\mathrm{c}$ separation is the vertex mass. If no displaced vertex has been associated to the jet, we look for tracks with large impact parameters. The impact parameter significance in $r-\phi$ and $\mathrm{Z}$ of the first and second most significant tracks of the jet are important input variables. In total, the BDTs are using 20 to 30 input variables. The output of the BDTs is the probability that the jet stems from a b or a c-quark decay. The LCFIPlus has succeeded the LCFIVertex flavour tagging, which was based on neural nets.

The ILD flavour tagging abilities have been studied using fully simulated and reconstructed samples of $Z \rightarrow q \bar{q}$ at $\sqrt{S}=91$ and $250 \mathrm{GeV}$ and of $Z \rightarrow q \bar{q} q \bar{q} q \bar{q}$ at $\sqrt{S}=500 \mathrm{GeV}$ and $1 \mathrm{TeV}$, where $Z$, in the latter case, is decaying to the same quark pairs. The beam background was not taken into consideration for this study. ILD performance is illustrated in Figure 6.

The ILD flavour tagging in the presence of the beam background is currently under study. Using the standard tracking software, its performance is highly degraded: a steep drop in b-tagging purity by $52.4 \%$ (for $80 \%$ of efficiency) and in c-tagging purity by $36.4 \%$ (for $60 \%$ of efficiency) 

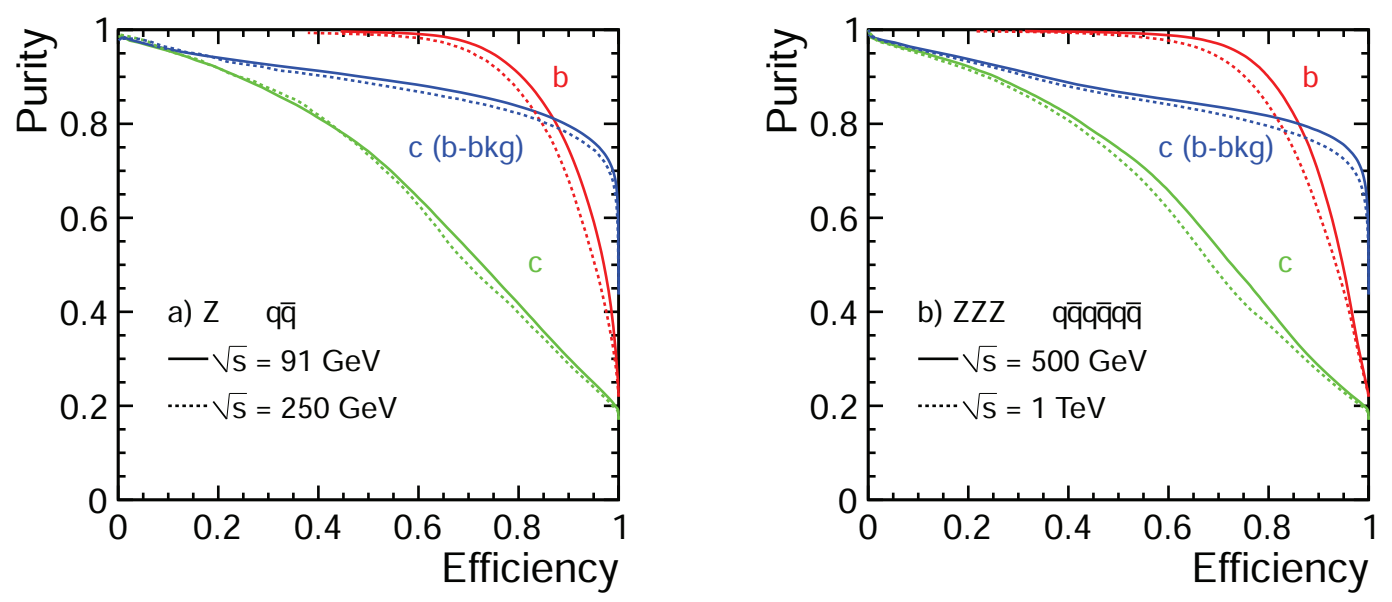

Figure 6: ILD flavour tagging performance. In the right plot, we can see purity versus efficiency for b- and c-tagged jets for $Z \rightarrow q \bar{q}$ at $\sqrt{S}=91$ and $250 \mathrm{GeV}$. In the left plot, we can see purity versus efficiency for b- and c-tagged jets for $Z \rightarrow q \bar{q} q \bar{q} q \bar{q}$ at $\sqrt{S}=500 \mathrm{GeV}$ and $1 \mathrm{TeV}$. Blue colour: purity versus efficiency for c-tagged jets when only b quarks have been taken as background.

has been observed. Replacing the standard standalone silicon tracking with the FPCCD track finder, significantly better results have been obtained: the degradation in b-tagging and c-tagging purity, in the presence of the beam background, is $5.2 \%$ and $7 \%$ respectively [12].

The SiD flavour tagging abilities have been studied inside the framework of the Higgs branching ratios and the $t \bar{t} h$ analysis. Figure 7 shows the b-tagging efficiency of a b-quark sample versus the b-tagging efficiency of a light (uds) and a c-quark sample. In the right plot, beam background (pairs and $\gamma \gamma \rightarrow$ hadrons) corresponding to one bunch crossing has been overlaid.
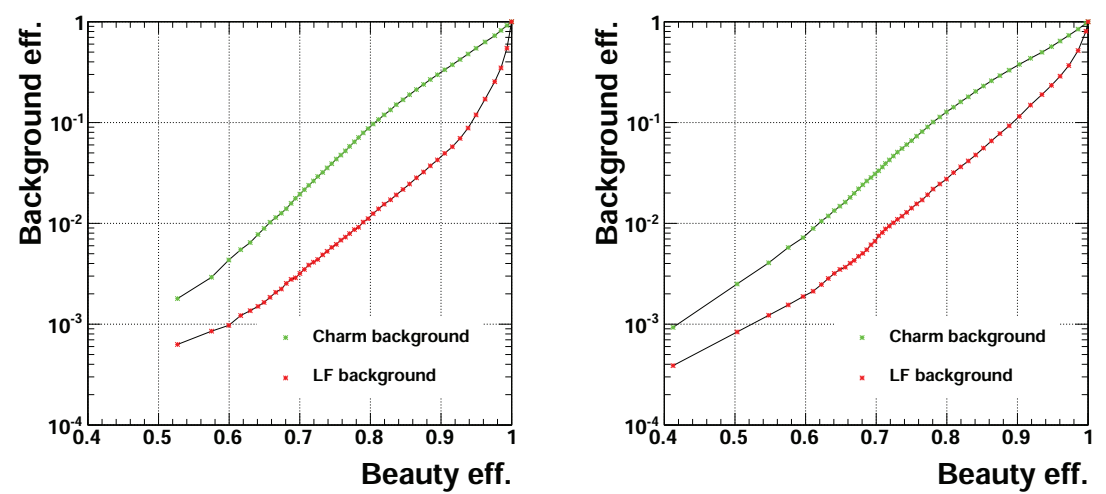

Figure 7: Mis-identification efficiency of a light (red markers) and a c-quark sample (green markers) as beauty quarks against beauty identification efficiency. Left without beam background; right, when beam background corresponding to one bunch crossing has been overlaid.

\section{Conclusion}

Excellent vertexing performance is essential for the precision measurements foreseen for the ILC physics program. The realisation of this performance is based on the detector performance as 
well as on the maturity of the software tools. In this proceedings we reviewed the tools for tracking, vertexing and flavour tagging of the ILC. The feasibility of analyses in particular demanding on the performance of the above tools, like the Higgs hadronic branching ratios and the top Yukawa coupling, in a high level of realism, has been shown via full simulation studies in both detector's DBD. The goal of the on-going work is to fully understand the beam background effects on tracking, vertexing and flavour tagging performance.

\section{References}

[1] T. Behnke, J.E. Brau, P.N. Burrows, J. Fuster, M. Peskin, M. Stanitzki, Y. Sugimoto, S. Yamada, H. Yamamoto, The International Linear Collider Technical Design Report-Volume 4: Detectors, arXiv:1306.6329 [physics.ins-det].

[2] A. Vogel, Beam-Induced Backgrounds in Detectors at the ILC, PhD thesis, Hamburg University, 2008.

[3] F. Gaede et al, Track reconstruction at the ILC: the ILD tracking software, in proceedings of 20th International Conference on Computing in High Energy and Nuclear Physics (CHEP2013), Journal of Physics, 513 (2014) 022011

[4] T. Mori et al, Study of tracking and flavor tagging with FPCCD vertex detector, in proceedings of International Workshop on Future Linear Colliders, (LCWS13), November 2013, Tokyo, Japan, arXiv:1403.5659 [physics.ins-det]

[5] M. Winter et al, Development of CMOS Pixel Sensors fully adapted to the ILD Vertex Detector Requirements, in proceedings of 2011 International Workshop on Future Linear Colliders, Granada, Spain, 26-30 September 2011, arXiv:1203.3750 [physics.ins-det].

[6] R. Glattauer, Track reconstruction in the forward region of the detector ILD at the electron-positron linear collider ILC, Master Thesis, Vienna University of Technology, 2012

[7] D. Onoprienko, E. von Toerne, Calorimeter Assisted Tracking Algorithm for SiD, arXiv:0711.0134 [physics.ins-det].

[8] D. Bailey et al, The LCFIVertex package: vertexing, flavour tagging and vertex charge reconstruction with an ILC vertex detector, NIM A $\mathbf{6 1 0}$ (573) 2009.

[9] T. Tanabe and T. Suehara, https://confluence.slac.stanford.edu/display/ilc/LCFIPlus, 2013.

[10] D.J. Jackson, A Topological Vertex Reconstruction Algorithm for Hadronic Jets, NIM A 388 (247) 1997.

[11] E. Devetak, Measurement of the Top Quark Mass, Cross Section and Anomalous Couplings at the International Linear Collider, $\mathrm{PhD}$ thesis, University of Oxford, 2009

[12] J. Strube, FPCCD Flavor Tagging, talk in Americas Workshop on Linear Colliders, May 2014, Fermilab, USA. 